\title{
BMJ Open Ultrasound measurements of pathological and physiological skin thickness: a scoping review protocol
}

\author{
Brandon Meikle (D) , ${ }^{1,2}$ Roy M Kimble, ${ }^{3,4}$ Zephanie Tyack (D) ${ }^{2,5}$
}

To cite: Meikle B, Kimble RM, Tyack Z. Ultrasound measurements of pathological and physiological skin thickness: a scoping review protocol. BMJ Open 2022;12:e056720. doi:10.1136/ bmjopen-2021-056720

- Prepublication history and additional supplemental material for this paper are available online. To view these files, please visit the journal online (http://dx.doi.org/10.1136/ bmjopen-2021-056720).

Received 25 August 2021 Accepted 11 January 2022

D) Check for updates

(c) Author(s) (or their employer(s)) 2022. Re-use permitted under CC BY-NC. No commercial re-use. See rights and permissions. Published by BMJ.

For numbered affiliations see end of article.

\section{Correspondence to} Mr Brandon Meikle; brandon.meikle@uqconnect. edu.au

\section{ABSTRACT}

Introduction Ultrasound is a quick, safe, and noninvasive imaging method that can be used to measure skin thickness in pathological cutaneous conditions in clinical and research settings. Despite widespread use, there exists a lack of standardisation and reporting of ultrasound skin thickness measurement methods, which makes between-studies comparisons difficult. To address this, we present a scoping review protocol, which aims to determine what is and is not known about the measurement of skin and scar thickness using ultrasound in people with traumatic scars.

Methods and analysis The Preferred Reporting Items for Systematic Reviews and Meta-Analyses extension for Scoping Reviews guidelines and Joanna Briggs Institute scoping review methodology will be used to guide this review. Electronic database searching will be conducted in Ovid MEDLINE, Embase, Cumulative Index of Nursing and Allied Health Literature and Web of Science. No date limit will be imposed on the database searches. Records will be supplemented with searches of reference lists of included studies and grey literature in OpenGrey and Google Advanced. Screening will be conducted by two independent reviewers, and studies where ultrasound is used to measure skin and scar thickness in people with traumatic scars will be included. Data extraction will include ultrasound methods (eg, transducer orientation), psychometric properties (eg, reliability, measurement error), health service and implementation outcomes (eg, feasibility, acceptability) and factors influencing ultrasound measurement of skin thickness (eg, body location, age).

Ethics and dissemination Ethical approval is not required for this investigation, as published literature will form the basis of the review. The review will be published in a peer-reviewed scientific journal and is expected to result in the development of the first evidence-based and consensus-based methodological guideline for skin thickness measurement by ultrasound.

\section{INTRODUCTION}

Measurement of skin thickness is important for the diagnosis and monitoring of skin physiology and pathology in clinical practice, and is a commonly reported outcome in skin research. ${ }^{12}$ Applications of ultrasound measurement of skin thickness include measurement of pathological changes to skin thickness in conditions such as scleroderma

\section{Strengths and limitations of this study}

- This scoping review will map methods used in the literature to measure skin and scar thickness by ultrasound in people with traumatic scars, which has not been previously conducted.

- The protocol includes structured searches of four electronic databases of peer-reviewed literature, complemented by searches of grey literature repositories and handsearching of reference lists to identify a broad range of literature.

- It is anticipated that the results of the review will be used to inform a consensus-based methodological guideline.

- Due to the nonstandard nature of ultrasound methods reporting in published literature, it is possible that some relevant publications may not be identified by the literature search.

- Psychometric properties, implementation outcomes and measurement error of cutaneous ultrasound measurements will be reported, where available, to inform the most appropriate measurement methods and processes.

and scarring, as well as changes arising from natural and sun-affected ageing. ${ }^{3-5}$ In addition to pathological skin conditions, measurement of physiological, 'healthy' or 'normal' skin is often used in research, either as controls for measurement of pathologically affected skin, or in areas such as the development of intradermal vaccine delivery ${ }^{6}$ or improving insulin delivery as therapy for diabetics. ${ }^{5} 7$ With such a wide range of potential applications, quantifying skin thickness has become an important part of both routine medical care and research. ${ }^{8-10}$

The first use of ultrasound to measure skin thickness occurred in 1979 using A-mode (amplitude mode) ultrasound. ${ }^{11}$ Since then, B-mode (brightness) ultrasound has become the predominant form of medical ultrasonography. The difference between these two modes is seen through their presentation of ultrasound data, with A-mode showing data as peaks on an amplitude-time graph, and 
B-mode ultrasound producing black-and-white crosssectional images of the tissues underlying the transducer. ${ }^{12} \mathrm{~B}$-mode images translate the amplitude-time data of A-mode ultrasound into pixel brightness and spatial information respectively. ${ }^{12}$ For the purpose of skin and scar thickness measurement, B-mode ultrasound has advantages as it reduces the ambiguity of measurement between tissue interfaces compared with A-mode ultrasound. ${ }^{12} 13$

B-mode devices also hold other advantages for medical use when compared with A-mode ultrasound. One advantage is that they are generally capable of varying the frequency of emitted sound, allowing for the generation of high-frequency ultrasound (ie, $>20 \mathrm{MHz}$ ). Highfrequency ultrasound is useful for skin and scar thickness measurement, as it provides high resolution imaging of superficial structures, functions and pathologies, allowing for more accurate distinctions to be made between, for example, the epidermis and dermis. This improved high resolution imaging is provided, however, at the expense of the ability to visualise deeper structures. ${ }^{13-15}$ B-mode ultrasound also forms the basis of other ultrasound methods such as Doppler or elastography, where additional information, such as blood flow or tissue elasticity, respectively, is presented over a B-mode image. ${ }^{11} 1617$ The versatility of ultrasound, alongside its portability and ease of use have seen it become increasingly popular with clinicians and researchers investigating the skin. ${ }^{11} 18$ A novel form of ultrasound, ultra-high frequency ultrasound, allows measurement between 30 and $100 \mathrm{MHz}$, and is currently being evaluated for use in dermatological conditions, with promising results. ${ }^{19}$

Ultrasound is a medical imaging method that permits the measurement of skin and scar thickness safely and non-invasively, while being both time-effective and costeffective. $^{13}$ B-mode ultrasound has many additional benefits as a measurement instrument of in vivo skin and scar thickness, particularly when compared with other medical imaging modalities such as X-ray, MRI and CT scans. These advantages include its portability, fast 'realtime' image capture, not requiring extended periods of patient immobilisation, and not using ionising radiation, all of which support its versatile use at patient bedsides, in outpatient clinics and in research. ${ }^{132021}$ The speed of the ultrasound measurement is particularly advantageous for its use in paediatric populations, as patients are not required to stay still for extended periods of time, minimising the need for sedatives, general anaesthetics or other immobilising agents. ${ }^{172022} 23$

Despite being a largely objective measure, the capture and analysis of ultrasound images for cutaneous thickness measurement can be considered subjective, as these steps are both operator-dependent and situationdependent. ${ }^{1} 172425$ Examples of the methodological variation inherent in ultrasound measurement include transducer placement and orientation, ultrasound frequency and methods to prevent skin compression. Alongside the physical act of image acquisition, image analysis is also user-dependent, particularly where ambiguity exists distinguishing borders of various structures. In the context of ultrasound measurements of skin thickness, this is shown through the requirement that the structures and layers of the skin are correctly identified each time. This is particularly important where pathology or treatment has altered one or more layers of the skin from physiological 'normal', changing its appearance on the ultrasound image, such as in traumatic scarring. ${ }^{24}$ Considering methodological variations is also important when undertaking inter-study comparisons, or when attempting to minimise or investigate ultrasound error, thus the need for a standardised methodological protocol. At present, such a protocol does not yet exist. Despite reported measurement error being low within individual studies, ${ }^{2326}$ the lack of reporting or standardisation means that this assumption cannot be broadened to interstudy comparisons or meta-analyses. The importance of standardisation of ultrasound measurement has previously been recognised by the Dermatologic Ultrasound (DERMUS) group, who created a consensus-based ultrasound methodology for the measurement of skin lesions. ${ }^{15}$ This guide provides a good starting point, however, little guidance is given for the explicit measurement of skin or scar thickness with ultrasound. ${ }^{15}$ We aim to address this gap, focusing on skin and scar thickness measurements in people with traumatic scars. Traumatic scarring is defined as arising following injury to the skin caused by sharp object penetration (including as a result of surgery or vaccination) or burns. ${ }^{27}$

To improve the reporting, reproducibility and generalisability of skin and scar thickness measurements taken by ultrasound, we aim to first conduct a scoping review of the literature, which we anticipate will identify wide variability in these methods. We intend to use these findings to inform a consensus-based methodological guideline for the measurement of skin and scar thickness by ultrasound. We anticipate that studies and meta-analyses that use the guideline may improve the quality of results, methodological reporting standards and ability to make comparisons between studies.

\section{METHODS AND ANALYSIS \\ Protocol design}

This protocol is designed following the recommendations of the Joanna Briggs Institute (JBI) ${ }^{28}$ and reported according to the Preferred Reporting Items for Systematic Reviews and Meta-Analyses extension for Scoping Reviews (PRISMA-ScR) ${ }^{29}{ }^{30}$ The protocol for the review is outlined in four steps: (1) identifying the research questions; (2) identifying relevant literature; (3) collecting data; and (4) analysing and synthesising results.

\section{Step 1: Identifying the research questions}

To understand the variability present in ultrasound measurements of skin thickness, the following overarching research question was posed: 'What do we know and 
not know about the measurement of skin and cutaneous scar thickness in people with traumatic scarring using ultrasound?'. To further expand this question, the literature will be reviewed using the following subquestions:

1. 'What are the methods that have been used to measure skin and scar thickness by ultrasound?'

2. 'What are the psychometric properties reported for ultrasound in the context of skin and scar thickness measurement?'

3. 'What is the feasibility and clinical, health service and implementation outcomes related to ultrasound measurement of skin and scar thickness?'

4. 'What are the factors considered and influencing the measurement of skin and scar thickness using ultrasound'?

5. 'What are the strengths and limitations of measurement methods?

6. 'What guidelines or frameworks have been used to inform measurement techniques or instrument selection?'

\section{Step 2: Identifying relevant literature}

Search strategy

Electronic databases will be searched to identify published, peer reviewed literature using a standardised search strategy centred around four themes: 'ultrasound', 'skin', 'thickness' and 'measure'. This search will be run in Ovid MEDLINE, Embase, Cumulative Index of Nursing and Allied Health Literature (CINAHL) and Web of Science. No date limit will be imposed in the databases during the search. The search strategy was initially developed for Ovid MEDLINE (box 1) with the assistance of a faculty librarian, and modified for the other databases (online supplemental material 1) using the online 'Polyglot Search Translator', which automatically adapts the syntax of the search for different databases. ${ }^{31}$ The efficacy

\section{Box 1 Search strategy developed for Ovid MEDLINE}

((ultrasound.ti,ab. OR ultra sound.ti,ab. OR sonograph*.ti,ab. OR ultrasonic.ti,ab. OR high-frequency.ti,ab. OR high frequency.ti,ab. OR hfus. ti,ab. OR ultrasonog*.ti,ab. OR exp Ultrasonography/)

AND

(skin.ti,ab. OR epiderm*.ti,ab. OR derm*.ti,ab. OR cutaneous.ti,ab OR scar*.ti,ab OR keloid*.ti,ab OR cicatri*.ti,ab OR exp Skin/ OR exp Dermatology/ OR exp Cicatrix/)

AND

((thickness*.ti,ab. OR thicken*.ti,ab. OR depth.ti,ab. OR volume.ti,ab. OR height.ti,ab. OR vancouver scar scale.ti,ab)

ADJ10

(measure ${ }^{\star} . t i, a b .0 R$ quantif*.ti,ab. OR calculat*.ti,ab OR estimat*.ti,ab OR assess*.ti,ab. OR ncluding*.ti,ab. OR evaluat*.ti,ab OR imag*.ti,ab OR exam*.ti,ab)))

NOT (exp animals/ NOT exp humans/)

ab, abstract (searches the abstract of the publication); adj10, adjacency (search terms must be located within 10 words of one another); exp, explode (used for MeSH headings to include all subheadings); ti, title (searches the title of the publication). of the search strategy in identifying appropriate publications was tested by determining how well the search was able to identify a sample of 19 peer-reviewed publications known to the authors.

The nature of this methodology-centric review warrants the use of additional search strategies and techniques. Reference lists of records that progress to full-text review will also be searched to identify any further publications that may be relevant. To identify non peer-reviewed grey literature sources such as ultrasound manufacturer's guidelines, course notes and theses, searching will be conducted using the phrase 'ultrasound skin thickness measurement' in OpenGrey, Google Scholar and Google Advanced. Searches conducted in Google Scholar and Google Advanced will be limited to the first 200 results. $^{32}$

Search results will be screened by at least two independent researchers using Covidence systematic review software (Veritas Health Innovation, Melbourne, Australia. Available at www.covidence.org). As detailed methodologies are rarely reported in the abstract, it is anticipated that many publications will progress from title/abstract screening to full-text review. Disagreements will be resolved through discussion with a third author.

\section{Inclusion criteria}

The inclusion criteria for relevant literature are based around the overarching primary research question using the population concept context (PCC) mnemonic as outlined by the JBI (table 1$).{ }^{28}$ Identified publications will be grouped into adult and paediatric samples for analysis and dissemination.

\section{Types of sources}

Original research, including randomised controlled trials, case studies involving any number of participants and clinical and/or methodological guidelines, will be eligible for inclusion. Grey literature sources, including theses, manufacturer guidelines and course notes will also be included. Reviews, discussion papers and opinion pieces will not be included but may be used to inform the interpretation of the results. Where identified by the search, the reference lists of included records will be searched for additional records. Search records and screening will be reported using a PRISMA flow diagram (figure 1). ${ }^{33}$

Exclusion criteria:

Records that use ultrasound to measure the thickness of non-traumatic scars (ie, those not caused by sharp injuries or burns) will not be included in this review. We will also not include records where ultrasound is used to measure skin thickness in non-traumatic conditions, such as scleroderma or diabetes, or in traumatic conditions such as traumatic brain injury, where there is no cutaneous scarring. Records that use A-mode ultrasound to measure skin or scar thickness will also be excluded. 
Table 1 Review inclusion criteria mapped to the population, concept and context (PCC) mnemonic outlined by the Joanna Briggs Institute ${ }^{28}$

\begin{tabular}{ll} 
PCC element & Inclusion criteria \\
\hline Population & $\begin{array}{l}\text { Publications that use ultrasound to measure } \\
\text { skin or scar thickness on living, human } \\
\text { individuals with traumatic scars (arising } \\
\text { from penetration of the skin with sharp } \\
\text { objects including surgery or vaccination } \\
\text { or as a result of burns, including thermal, } \\
\text { chemical or friction). } \\
\text { No age limit or limit on other } \\
\text { sociodemographic factors }\end{array}$ \\
& $\begin{array}{l}\text { Use of B-mode or ultra-high frequency } \\
\text { ultrasound to measure skin or scar } \\
\text { thickness }\end{array}$ \\
& $\begin{array}{l}\text { Any clinical or research settings where } \\
\text { ultrasound is used to measure scar or skin } \\
\text { thickness }\end{array}$ \\
\hline
\end{tabular}

\section{Step 3: Collecting the data}

Data from included studies will be extracted into an extraction spreadsheet in Microsoft Excel. Data extraction will be completed by one author and checked by another author. Discrepancies or disagreements will be resolved through discussion.

General information collected from each publication will include author name(s), and year and country of publication. Publication-specific information collected will include: number of participants, nature of the investigation (ie, clinical measurement or research outcomes), 'condition' (eg, pathological skin condition or physiological skin measurement) and publication type (eg, original research, clinical guideline). Demographic information relating to the participants from each study will also be included: age, gender and other conditions or comorbidities. Finally, the specific methods used to measure skin and scar thickness using ultrasound will be reviewed to answer the research questions (table 2). Where reported, psychometric properties of the ultrasound methods will be reported using the COSMIN Risk of Bias tool to assess the quality of studies on reliability or measurement error of outcome measurement instruments (eg, reliability, reproducibility, measurement error, minimal clinically important differences, validity), ${ }^{34}$ alongside feasibility outcomes based on Prinsen et $a l^{35}$ (eg, cost of instrument, time taken for measurement, availability of the instrument, ease of administration, number of steps and personnel involved, considerations for special populations such as young children).$^{35}$ Clinical, health service or implementation outcomes (other than feasibility related to the ultrasound measurement of scar and skin thickness), will be reported in accordance with Proctor et $a \hat{l}^{36}$, namely: acceptability, adoption, appropriateness, fidelity, implementation cost, penetration and sustainability. ${ }^{36}$ Although included in these implementation outcomes, feasibility will not be assessed according to Proctor et al. ${ }^{36}$ Instead, this outcome will be assessed using the measurement instrument-specific feasibility aspects outlined by Prinsen et al: ${ }^{35}$ ease of administration, completion time (ie, how long the ultrasound measurement

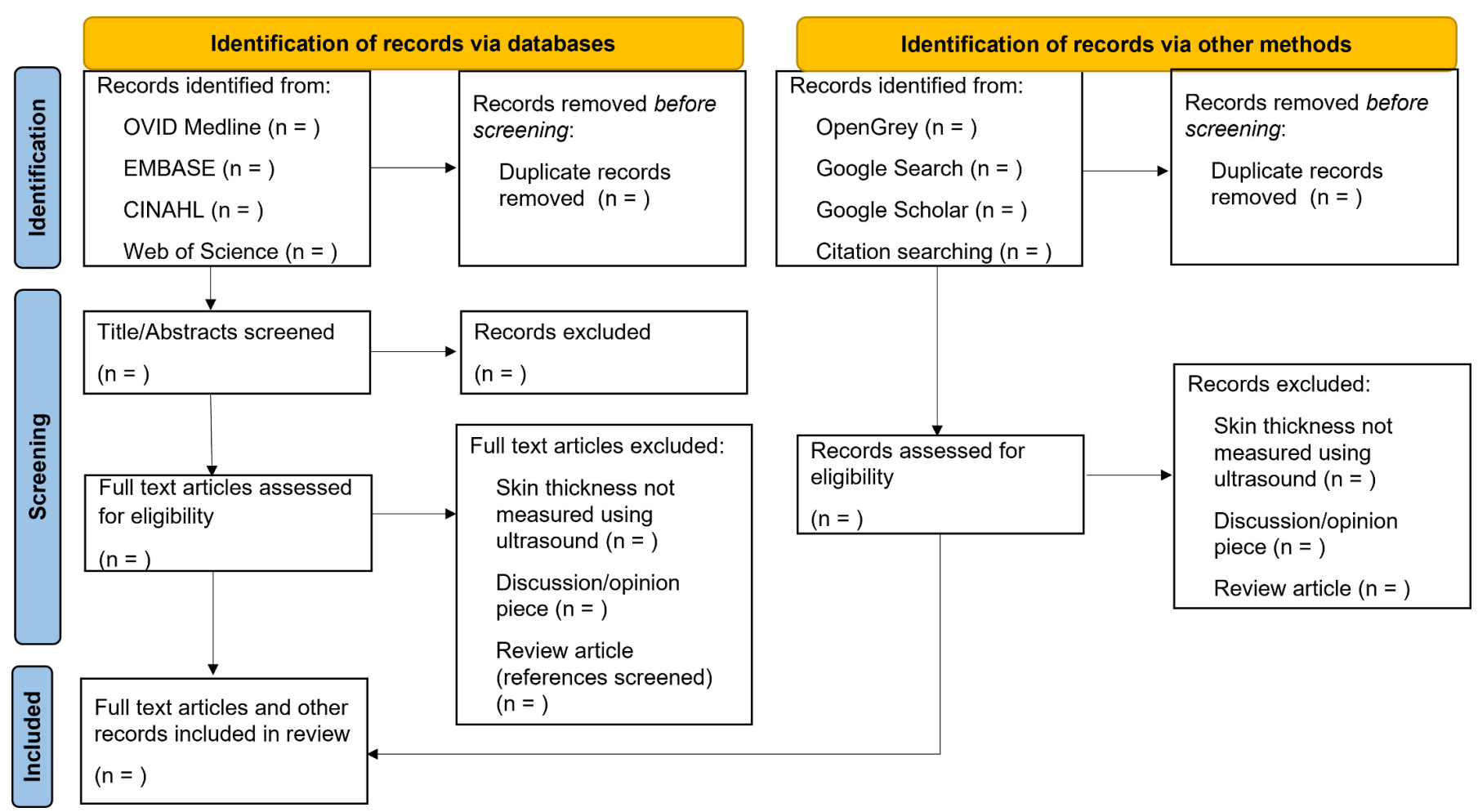

Figure 1 Preferred Reporting for Systematic Reviews and Meta-Analyses flow diagram for the scoping review process. Modified from Page et al. ${ }^{33}$ 
Table 2 Data extraction fields mapped to scoping review research questions

\begin{tabular}{|c|c|}
\hline Research question & Data extraction field \\
\hline \multirow{8}{*}{$\begin{array}{l}\text { What are the methods that have been used to measure skin } \\
\text { and scar thickness by ultrasound? }\end{array}$} & Ultrasound device name \\
\hline & Ultrasound frequency \\
\hline & Anatomical locations/functional measurement units measured \\
\hline & Patient orientation \\
\hline & Transducer orientation \\
\hline & Methods used to prevent skin compression \\
\hline & Measurement site relocation \\
\hline & $\begin{array}{l}\text { Type of measurement (eg, epidermis/dermis/combined) } \\
\text { Measurement of contralateral or unaffected skin for comparis }\end{array}$ \\
\hline
\end{tabular}

What are the psychometric properties reported for ultrasound Reliability in the context of skin and scar thickness measurement?*

Reproducibility

Measurement error

Minimal clinically important difference

\begin{tabular}{|c|c|}
\hline $\begin{array}{l}\text { What is the feasibility } † \text { and clinical, health service and } \\
\text { implementation outcomes related to ultrasound measurement } \\
\text { of skin and scar thickness? } \ddagger\end{array}$ & $\begin{array}{l}\text { Acceptability } \\
\text { Adoption } \\
\text { Appropriateness } \\
\text { Fidelity } \\
\text { Implementation cost } \\
\text { Penetration } \\
\text { Sustainability } \\
\text { Instrument cost } \\
\text { Time taken for measurement } \\
\text { Instrument availability } \\
\text { Ease of administration } \\
\text { No of steps/personnel involved } \\
\text { Considerations for special populations }\end{array}$ \\
\hline $\begin{array}{l}\text { What are the strengths and limitations of the measurement } \\
\text { methods? }\end{array}$ & Reported strengths/limitations of method \\
\hline $\begin{array}{l}\text { What guidelines or frameworks have been used to inform } \\
\text { measurement techniques or instrument selection? }\end{array}$ & Guidelines/frameworks used \\
\hline
\end{tabular}

${ }^{*}$ Psychometric properties as per the COSMIN Risk of Bias tool to assess the quality of studies on reliability or measurement error of outcome measurement instruments ${ }^{34}$

†Feasibility outcomes as per Prinsen et al. ${ }^{35}$

†Implementation outcomes as per Proctor et al. ${ }^{36}$

took), ease of standardisation, cost, required instrument, availability in different settings and ease of score calculation. $^{35}$ Reliability and measurement error will be reported using the COSMIN Risk of Bias tool for outcome measurement instruments. ${ }^{34}$

\section{Step 4: Analysing and synthesising the results}

Findings will be synthesised and summarised descriptively, in summary tables, or diagrammatically where appropriate. Critical appraisal of individual studies will not be undertaken, as the focus of this investigation is to map the methods in which ultrasound has been used to measure skin thickness, not to assess the quality of outcomes or studies. It is expected that, in many cases, ultrasound will only comprise a small part of the larger study, and therefore, have minimal bearing on the quality of the overall publication. 


\section{Patient and public involvement}

There is no patient and public involvement in the design, conduct, reporting or dissemination of this research.

\section{Ethics and dissemination}

This scoping review will, to the best of our knowledge, be the first to systematically map the evidence regarding the measurement of traumatic scar thickness using ultrasound. It is anticipated that the results of the scoping review will inform a consensus-based methodological guideline that will have broad applicability to scar measurement in both clinical practice and research. As an example, this guideline will likely be implemented in routine clinical practice for the measurement of hypertrophic burn scars at the Pegg Leditschke Children's Burns Centre at the Queensland Children's Hospital, a major metropolitan paediatric hospital located in Brisbane, Australia.

The methodology of this proposed scoping review will consist of accessing, reviewing and analysing data from published literature. As such, institutional ethics approval is not required.

\section{Author affiliations}

${ }^{1}$ Children's Health Research Centre, The University of Queensland, Faculty of Medicine and Biomedical Sciences, Herston, Queensland, Australia

${ }^{2}$ Centre for Children's Burns and Trauma Research, The University of Queensland, South Brisbane, Queensland, Australia

${ }^{3}$ Queensland Children's Hospital, Centre for Children's Burns \& Trauma Research, Brisbane, Queensland, Australia

${ }^{4}$ Pegg Leditshke Children's Burns Centre, Children's Health Queensland Hospital and Health Service, South Brisbane, Queensland, Australia

${ }^{5}$ Australian Centre for Health Service Innovation (AusHSI), Centre for Healthcare Transformation, and School of Public Health and Social Work, Queensland University of Technology, Brisbane, Queensland, Australia

\section{Twitter Brandon Meikle @BrandonMeikle5 and Zephanie Tyack @tyack_z}

Acknowledgements The authors would like to acknowledge faculty librarian, Mr David Honeyman, for his invaluable contribution assisting in the preparing the search strategy.

Contributors $\mathrm{BM}$ and $\mathrm{ZT}$ conceived the project after identifying this area as a knowledge gap in existing literature. BM developed the research questions and study methodology, under the guidance of ZT. RMK and ZT contributed to the supervision of $\mathrm{BM}$ as a PhD student. BM drafted the paper, and $\mathrm{ZT}$ and RK provided critical appraisal of the drafted manuscript.

Funding This research received no funding from any agency in the public, commercial or not-for-profit sectors. BM is a recipient of a Research Training Program Scholarship awarded by The University of Queensland, where he is a current PhD candidate.

Competing interests None declared.

Patient consent for publication Not applicable.

Provenance and peer review Not commissioned; externally peer reviewed.

Supplemental material This content has been supplied by the author(s). It has not been vetted by BMJ Publishing Group Limited (BMJ) and may not have been peer-reviewed. Any opinions or recommendations discussed are solely those of the author(s) and are not endorsed by BMJ. BMJ disclaims all liability and responsibility arising from any reliance placed on the content. Where the content includes any translated material, BMJ does not warrant the accuracy and reliability of the translations (including but not limited to local regulations, clinical guidelines, terminology, drug names and drug dosages), and is not responsible for any error and/or omissions arising from translation and adaptation or otherwise.

Open access This is an open access article distributed in accordance with the Creative Commons Attribution Non Commercial (CC BY-NC 4.0) license, which permits others to distribute, remix, adapt, build upon this work non-commercially, and license their derivative works on different terms, provided the original work is properly cited, appropriate credit is given, any changes made indicated, and the use is non-commercial. See: http://creativecommons.org/licenses/by-nc/4.0/.

ORCID iDs

Brandon Meikle http://orcid.org/0000-0002-3003-3589

Zephanie Tyack http://orcid.org/0000-0003-3376-5731

\section{REFERENCES}

1 Wortsman X, Alfageme F, Roustan G, et al. Guidelines for performing dermatologic ultrasound examinations by the DERMUS group. $J$ Ultrasound Med 2016;35:577-80.

2 Wortsman X. Common applications of dermatologic sonography. $J$ Ultrasound Med 2012;31:97-111.

3 Chen C, Cheng Y, Zhu X, et al. Ultrasound assessment of skin thickness and stiffness: the correlation with histology and clinical score in systemic sclerosis. Arthritis Res Ther 2020;22:1-197.

4 Berman B, Maderal A, Raphael B. Keloids and hypertrophic scars: pathophysiology, classification, and treatment. Dermatol Surg 2017;43 Suppl 1:S3-18.

5 Derraik JGB, Rademaker M, Cutfield WS, et al. Effects of age, gender, BMI, and anatomical site on skin thickness in children and adults with diabetes. PLoS One 2014;9:e86637-e.

6 Van Mulder TJS, de Koeijer M, Theeten $\mathrm{H}$, et al. High frequency ultrasound to assess skin thickness in healthy adults. Vaccine 2017;35:1810-5.

7 Lo Presti D, Ingegnosi C, Strauss K. Skin and subcutaneous thickness at injecting sites in children with diabetes: ultrasound findings and recommendations for giving injection: skin and SC thickness in children with diabetes. Pediatr Diabetes 2012;13:525-33.

8 Gaitini D. Introduction to Color Doppler Ultrasound of the Skin. In: Dermatologic ultrasound with clinical and histologic correlations. New York: Springer, 2013: 3-14.

9 Alsing KK, Serup J. High-Frequency ultrasound skin thickness: comparison of manual reading and automatic border detection includes assessment of interobserver variation of measurement. Skin Res Technol 2020;26:832-8.

$10 \mathrm{Li} \mathrm{H}$, Furst DE, Jin H, et al. High-Frequency ultrasound of the skin in systemic sclerosis: an exploratory study to examine correlation with disease activity and to define the minimally detectable difference. Arthritis Res Ther 2018;20:181.

11 Alexander H, Miller DL. Determining skin thickness with pulsed ultra sound. J Invest Dermatol 1979;72:17-19.

12 Bushberg JT, Seibert JA, Leidholdt EM. Chapter 14 - Ultrasound. In: The essential physics of medical imaging. 3rd edn. Philadelphia: Lippincott Williams \& Wilkins, 2012.

13 Introduction STL. Diagnostic ultrasound imaging: inside out. 2nd edn. United States of America: Academic Press, 2014.

14 Szabo TL. Imaging Systems and Applications. In: Diagnostic ultrasound imaging: inside out. 2nd edn. The United States of America: Elsevier Academic Press, 2014: 297-336.

15 Wortsman X. Technical Considerations of the Dermatologic Ultrasound Examination. In: Atlas of dermatologic ultrasound. Switzerland: Springer International Publishing AG, 2018.

16 Jasaitiene D, Valiukeviciene S, Linkeviciute G, et al. Principles of high-frequency ultrasonography for investigation of skin pathology. $J$ Eur Acad Dermatol Venereol 2011;25:375-82.

17 Rodríguez Bandera Al, Sebaratnam DF, Feito Rodríguez M, et al. Cutaneous ultrasound and its utility in pediatric dermatology. Part I: lumps, bumps, and inflammatory conditions. Pediatr Dermatol 2020;37:29-39.

18 Kleinerman R, Whang TB, Bard RL, et al. Ultrasound in dermatology: principles and applications. J Am Acad Dermatol 2012;67:478-87.

19 Izzetti R, Vitali S, Aringhieri G, et al. Ultra-High frequency ultrasound, a promising diagnostic technique: review of the literature and singlecenter experience. Can Assoc Radiol J 2021;72:418-31.

20 Hertzberg BS, Middleton WD. Practical Physics. In: Ultrasound: the requisites. 3rd edn. Philadelphia: The United States of America: Elsevier, 2016.

21 Bamber J, Tristam M, Ultrasound D. The physics of medical imaging. Bristol, England: IOP Publishing, 1988: 319-88.

22 Rodríguez Bandera Al, Sebaratnam DF, Feito Rodríguez M, et al. Cutaneous ultrasound and its utility in pediatric dermatology: part II-Developmental anomalies and vascular lesions. Pediatr Dermatol 2020;37:40-51.

23 Simons M, Kee EG, Kimble R, et al. Ultrasound is a reproducible and valid tool for measuring scar height in children with burn scars: 
a cross-sectional study of the psychometric properties and utility of the ultrasound and 3D camera. Burns 2017;43:993-1001.

24 Mlosek RK, Malinowska S. Ultrasound image of the skin, apparatus and imaging basics. J Ultrason 2013:13:212-21.

25 Verhaegen PDHM, van der Wal MBA, Middelkoop E, et al. Objective scar assessment tools: a Clinimetric appraisal. Plast Reconstr Surg 2011;127:1561-70.

26 Nedelec B, Correa JA, Rachelska G, et al. Quantitative measurement of hypertrophic scar: interrater reliability and concurrent validity. $J$ Burn Care Res 2008;29:501-11.

27 Jagdeo J, Shumaker PR. Traumatic scarring. JAMA Dermatol 2017;153:364.

28 Peters MDJ, Godfrey CM, Khalil H, et al. Guidance for conducting systematic scoping reviews. Int J Evid Based Healthc 2015;13:141-6.

29 Moher D, Shamseer L, Clarke M, et al. Preferred reporting items for systematic review and meta-analysis protocols (PRISMA-P) 2015 statement. Syst Rev 2015;4:148-60.

30 Tricco AC, Lillie E, Zarin W, et al. PRISMA extension for scoping reviews (PRISMA-ScR): checklist and explanation. Ann Intern Med 2018;169:467-73.
31 Clark JM, Sanders S, Carter M, et al. Improving the translation of search strategies using the Polyglot search Translator: a randomized controlled trial. J Med Libr Assoc 2020;108:195-207.

32 Haddaway NR, Collins AM, Coughlin D, et al. The role of Google scholar in evidence reviews and its applicability to grey literature searching. PLoS One 2015;10:e0138237-e.

33 Page MJ, McKenzie JE, Bossuyt PM, et al. The PRISMA 2020 statement: an updated guideline for reporting systematic reviews. $J$ Clin Epidemiol 2021;134:178-89.

34 Mokkink LB, Boers M, van der Vleuten CPM, et al. COSMIN risk of bias tool to assess the quality of studies on reliability or measurement error of outcome measurement instruments: a Delphi study. BMC Med Res Methodol 2020;20:1-13.

35 Prinsen CAC, Vohra S, Rose MR, et al. How to select outcome measurement instruments for outcomes included in a "Core Outcome Set" - a practical guideline. Trials 2016;17:1745-6215.

36 Proctor E, Silmere H, Raghavan R, et al. Outcomes for implementation research: conceptual distinctions, measurement challenges, and research agenda. Adm Policy Ment Health 2011;38:65-76. 